\title{
An On-Line Algorithm for Checkpoint Placement
}

\author{
Avi Ziv, Member, IEEE, and Jehoshua Bruck, Senior Member, IEEE
}

\begin{abstract}
Checkpointing enables us to reduce the time to recover from a fault by saving intermediate states of the program in a reliable storage. The length of the intervals between checkpoints affects the execution time of programs. On one hand, long intervals lead to long reprocessing time, while, on the other hand, too frequent checkpointing leads to high checkpointing overhead. In this paper, we present an on-line algorithm for placement of checkpoints. The algorithm uses knowledge of the current cost of a checkpoint when it decides whether or not to place a checkpoint. The total overhead of the execution time when the proposed algorithm is used is smaller than the overhead when fixed intervals are used. Although the proposed algorithm uses only on-line knowledge about the cost of checkpointing, its behavior is close to the off-line optimal algorithm that uses a complete knowledge of checkpointing cost.
\end{abstract}

Index Terms-Fault-tolerant computing, checkpointing, on-line algorithm, performance optimization.

\section{INTRODUCTION}

C HECKPOINTING is a common technique for reducing the execution time of programs in the presence of faults. Checkpointing consists of saving intermediate states of the task in a reliable storage, and, upon a detection of a fault, restoring the previous stored state. Hence, checkpointing enables to reduce the time to recover from a fault, while minimizing the lost processing time.

The interval between checkpoints affects the execution time of a program. On one hand, inserting more checkpoints, and reducing the interval between checkpoints, reduces the reprocessing time after failures. On the other hand, checkpoints have checkpointing costs associated with them, and, therefore, inserting more checkpoints increases the overall checkpointing cost and the program execution time. This trade-off between the reprocessing time and the checkpointing overhead leads to an optimal checkpoint placement strategy that optimizes certain performance measures [3], [4], [5], [7].

Considerable theoretical work has been devoted to analyzing checkpointing schemes and determining the optimal checkpoint placement strategy. Brock [1] and Duda [4] analyzed the execution time of a program with and without checkpoints. Gelenbe [5] showed that, to maximize availability in transactions systems, checkpoint intervals should be deterministic and of the same length. L'Ecuyer and Malenfant [8] derived a numerical approach for availability in dynamic checkpointing strategies when the fault rate is not constant. Nicola and van Spanje [11] compared analysis and optimization of several checkpointing models that differ in the checkpoints' placement and fault occurrence in transaction systems. Kulkarni, Nicola, and Trivedi [7] in-

- A. Ziv is with IBM Israel, Science and Technology, MATAM-Advanced Technology Center, Haifa 31905, Israel. E-mail: aziv@vnet.ibm.com.

- J. Bruck is with the California Institute of Technology, Mail Code 136-93, Pasadena, CA 91125. E-mail: bruck@paradise.caltech.edu.

For information on obtaining reprints of this article, please send e-mail to: tc@computer.org, and reference IEEECS Log Number 105212. vestigated the effects of checkpointing on the execution time of a program in queueing systems. Coffman and Gilbert [3] described optimal strategies for placement of checkpoints in a single program. A good survey on checkpointing, describing the above work, can be found in [10].

In all the work described above, it is assumed that the checkpointing overhead does not depend on the time the checkpoint is taken. Another approach for placing checkpoints, that takes into account the change of checkpointing overhead over time, can be found in [2]. In that paper, Chandy and Ramamoorthy proposed an algorithm, based on a graph theoretic method, for a placement of checkpoints that allows the programmer to decide where to place checkpoints according to an a priori knowledge about the cost of checkpointing. In [12], Toueg and Babaoglu derive an algorithm for optimal placement of checkpoints when there is a small number of possible locations for the checkpoints and the cost of checkpointing and recovery at each such location is known. The CATCH tool [9] is a compiler assisted technique that helps to improve the placement of checkpoints using information about the cost of checkpointing that is gathered in previous executions of the program.

One of the operations that is performed at a checkpoint is saving the program state on a stable storage. Therefore, the size of the program state is one of the main factors that determine the checkpointing cost. During the execution of a program, the size of its state is dynamically changing due to allocation and deallocation of memory blocks. While the size of the program's state might not be known in advance, it is possible to keep track of the allocation and deallocation operations and to know the state size of the program at the current time. Therefore, an estimation of the checkpointing cost at the current time can be obtained.

In this paper, we present a new on-line algorithm for placement of checkpoints. The algorithm keeps track of the state size of the program, and uses it to estimate the cost of checkpointing at the current point of execution. The 
knowledge about the cost of checkpointing is used when deciding at which points in the program to place checkpoints.

The main idea in the algorithm described here is to look for points in the program in which placing a checkpoint is the most beneficiary. The algorithm tries to find points in the program in which the state size is small, and use these points for checkpoints. If such points are found, checkpoints are placed at these points with small intervals between the checkpoints, so that the reprocessing time after a fault is small. If no such point is found after a period of time, a checkpoint is taken at a point with higher cost to avoid long reprocessing time in case of a fault. The main difference between the adaptive checkpointing presented in [9] and the algorithm presented in this paper is that our algorithm not only looks for points with low checkpointing cost, it also changes the interval between checkpoints to fit the current cost of checkpointing.

We study the performance of the new on-line placement algorithm for the simple case when the program has only two possible state sizes. Comparison of the average execution time of a program when the proposed algorithm is used to the average execution time when the intervals between checkpoints are fixed shows that the overhead for the on-line algorithm is lower. Although the proposed algorithm uses only past and present information about the cost of checkpointing when deciding whether or not to place a checkpoint, its performance is close to the optimal placement strategy that knows the cost of all checkpoints ahead of time. Comparison of the decision on checkpoint placement done by the two algorithms shows that both algorithms can avoid long periods of high cost and efficiently use periods of low-cost checkpointing.

While the program might not know ahead of time how its state size is going to change, it can detect changes in the state size just before they occur. This additional knowledge can be used to further improve the placement algorithm. We show how the on-line algorithm can use this knowledge to place checkpoints just before the state size increases, and what benefits this knowledge can provide. Analysis of the modified algorithm shows that its performance is even closer to the optimal algorithm than the on-line algorithm.

The rest of the paper is organized as follows. In Section 2, we describe the model of the program and environment we use in this paper. In Section 3, we describe the new on-line algorithm. In Section 4, the performance of the new algorithm is compared with the fixed interval placement strategy and the optimal off-line placement strategy. In Section 5, a modification to the algorithm that enables to take advantage of detection of an increase in the state size before they occur is presented. In Section 6, we discuss some changes to the algorithm that make it fit a more realistic model better. Section 7 concludes the paper.

\section{BACKGROUND}

In this paper, we are interested in the average execution time of a program with checkpoints in a system that is vulnerable to faults, and the effects of different checkpointing placement strategies on the execution time. The faults in the system occur according to a Poisson process with rate $\lambda$. At some points during the execution of the program, checkpoints are placed. At each checkpoint, the state of the program is saved on a stable storage. After a fault is detected, the program is rolled back to the last saved state and execution is resumed from that point. We assume that the processor that executes the program detects faults immediately. We also assume that the time to roll the program back after a fault is detected is zero and that faults cannot occur during checkpointing.

While the assumptions that we make are not necessary for the operation of the algorithm presented in this paper, they make its analysis simpler and help to illustrate the advantages of the on-line algorithm. In Section 6, we examine what changes to the algorithm are needed to make it applicable to a more realistic environment.

Using these assumptions, we can calculate the average execution time of a program with faults and checkpoints. The analysis given here is the same one that is used by Duda in [4]. A program of length $t^{1}$ is divided into $n$ intervals of length $t_{1}, t_{2}, \ldots, t_{n}$, such that $\sum_{i=1}^{n} t_{i}=t$. At the end of each interval, a checkpoint is placed. The cost of the checkpoint at the end of the $i$ th interval is $c_{i}$. Let $T_{i}$ be the execution time of the ith interval, including the checkpointing time at the end of it. The $T_{i}$ s are random variables and their values depend on the number and locations of the faults that occur when the $i$ th interval is executed. The following proposition gives the average execution time of a single interval. The proof for the proposition can be found in [4].

Proposition 1. Under the assumptions stated above, $\bar{T}_{i}$, the average execution time of the ith interval and the overall execution time of the program are

$$
\bar{T}_{i}=\frac{e^{\lambda t_{i}}-1}{\lambda}+c_{i}, \quad \bar{T}=\sum_{i=1}^{n} \bar{T}_{i} .
$$

A good metric to measure the performance of a checkpointing placement strategy is the average overhead ratio $R$, which is defined as the ratio between the average overhead, caused by the checkpointing and the faults, and the program length. In other words,

$$
R=\frac{\bar{T}-t}{t}=\frac{\bar{T}}{t}-1
$$

When designing a placement strategy for checkpoints with a goal to minimize the overhead ratio $R$, two factors have to be considered: the overhead caused by the checkpoints themselves, and the reprocessing time that is needed after a fault is detected. If checkpoints are placed close to each other, then the reprocessing time after a fault has occurred is short. However, the overhead caused by the checkpoints themselves is high. When the checkpoints are far from each other, the checkpointing overhead is low, but long reprocessing might be needed after a fault is detected. An example on the effects of the interval length on the overhead ratio is shown in Fig. 1.

1. Throughout the paper, $t$, with and without subscripts, denotes productive time (i.e., excludes time spent in checkpointing, repair, recovery, and reprocessing) while $T$ denotes elapsed time. 


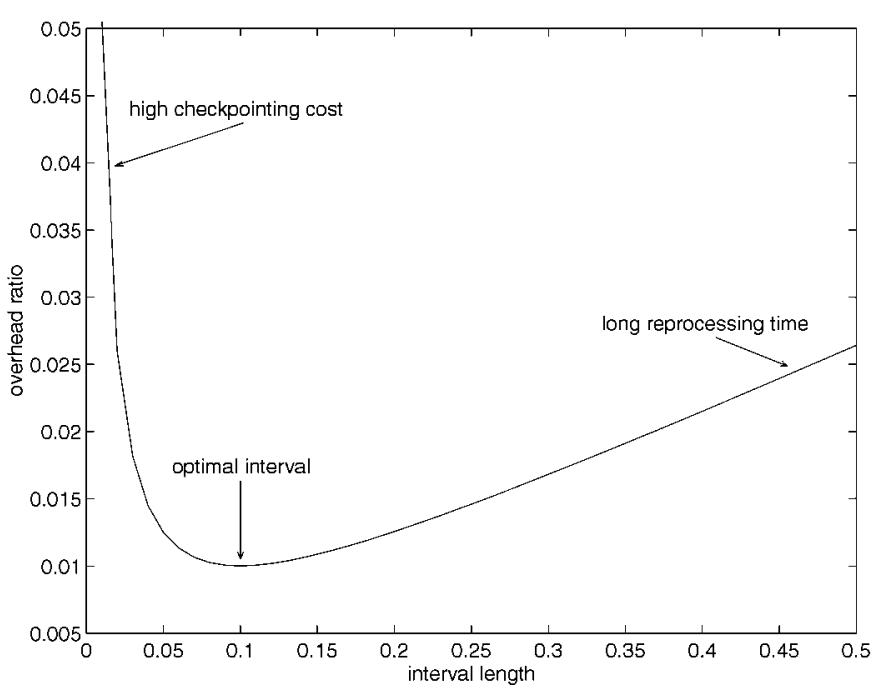

Fig. 1. Overhead ratio as a function of the interval length.

This trade-off between the checkpointing overhead and the reprocessing time leads to some optimal placement strategy that minimizes the overhead ratio $R$. This optimal placement strategy depends on the fault rate in the system and the cost of checkpointing. Next, we describe two existing placement strategies which we use for comparison with the on-line placement algorithm we present in this paper.

\subsection{Checkpointing with Fixed Intervals}

When the cost of checkpointing does not change with time, or when only the average cost is known, but not how it is changing with time, the optimal placement strategy is to place the checkpoints in fixed equidistant intervals [1], [4]. Because the execution times of different intervals are independent of each other when the location of the checkpoints are known, and because placing a checkpoint at a specific point does not effect future intervals, minimizing the overhead ratio for each interval alone leads to the optimal placement strategy. Since the checkpointing cost and the fault rate are the same during the execution, the optimal lengths of all the intervals are identical, and the optimal placement strategy is fixed equidistant intervals.

When fixed intervals are used, the overhead ratio of the whole program is the same as the overhead ratio for a single interval. Using Proposition 1, the overhead ratio for a single interval of length $t$ is

$$
R(t)=\frac{\bar{T}(t)}{t}-1=\frac{e^{\lambda t}+\lambda \bar{c}-1}{\lambda t}-1,
$$

where $\bar{c}$ is the average cost of a checkpoint. The optimal interval $\tilde{t}$ that minimizes the overhead ratio is roughly equal to $\tilde{t} \simeq \sqrt{\frac{2 \bar{c}}{\lambda}}[4]$.

Fig. 1 shows the overhead ratio as a function of the interval length when fixed intervals are used. In the figure, the fault rate is $\lambda=0.1$ and the cost of checkpointing is $c=$ 0.0005 . As can be seen in the figure, when $t=\sqrt{\frac{2 \bar{c}}{\lambda}}=0.1$, the overhead ratio is minimized.

\subsection{Optimal Placement Algorithm}

When checkpoints can be placed only in a finite number of locations and the cost of a checkpoint at each of these locations is known in advance, the optimal placement strategy can be found. In [12], Toueg and Babaoglu describe an algorithm for optimal checkpoints placement. In this algorithm, it is assumed that checkpoints can be placed only at a finite number of points in the program, and that the cost of checkpoints in each such point is known in advance. Using these assumptions, an $O\left(n^{2}\right)$ algorithm, based on dynamic programming technique, is given, where $n$ is the number of points where checkpoints can be placed.

\section{ON-LINE ALGORITHM FOR CHECKPOINTING Placement}

The checkpointing cost depends on the point in the program at which the checkpoint is placed. More specifically, the checkpointing cost depends on the size of the program's state at that point. Since the state size of the program changes during the execution due to memory allocation and deallocation operations, the checkpointing cost is changing with time according to some random process. Therefore, the fixed intervals placement strategy is not optimal. On the other hand, the state size of the program is usually not known in advance, and, therefore, the optimal off-line algorithm for placement of checkpoints is not practical.

While the state size of the program is not known in advance, the program can keep track of its state size by monitoring memory allocation and deallocation operations. By monitoring these operations, the program knows its current state size. Therefore, it can estimate the current cost of checkpointing. In this section, we show how knowledge about the current cost of checkpointing can be used in placement of checkpoints.

The main idea in the algorithm described here is to look for points in the program in which placing a checkpoint is the most beneficial. The algorithm monitors the state size of the program to find points in which the state size is small. If such points are found, checkpoints are placed at these points with small intervals between the checkpoints so that the reprocessing time after a fault is small. If no such point is found after a period of time, a checkpoint is placed at a point with higher cost to avoid long reprocessing time. In this case, the interval between the checkpoints is longer to reduce the checkpointing overhead.

To demonstrate how a current knowledge about the checkpointing cost can improve the performance of checkpointing schemes, we use the following example. The program has two possible state sizes, $s_{1}$ and $s_{2}$, such that $s_{1}<s_{2}$. The checkpointing cost when the state size is $s_{i}$ is $c_{i}\left(c_{1}<c_{2}\right)$. The state size of the program changes according to a two state Markov chain with rate of leaving state $s_{i}$ equal to $\mu_{i}$.

The algorithm works in the following way. We define two points in time, $t_{1}$ and $t_{2}$, such that $t_{1} \leq t_{2}$. The algorithm decides whether to place a checkpoint at $t$, where $t$ is the time since the last checkpoint, according to the following rules:

1) If $t<t_{1}$, don't place a checkpoint.

2) If $t_{1} \leq t<t_{2}$ and the state size is $s_{1}$, place a checkpoint at $t$. 
3) If $t=t_{2}$, place a checkpoint at $t$. The cost of the checkpoint is $c_{2}$.

Note that, in order to avoid high checkpointing overhead, a checkpoint is never placed before $t_{1}$. Also, to avoid long reprocessing time, a checkpoint is never placed after $t_{2}$. If, at some point $t \in\left[t_{1}, t_{2}\right)$, a small state size is found, then a checkpoint is placed at that point. Otherwise, a checkpoint is placed at $t_{2}$ with high cost. The values of $t_{1}$ and $t_{2}$ affect the performance of algorithm. By analyzing the overhead ratio of the algorithm, we can find the values of $t_{1}$ and $t_{2}$ that minimize the overhead ratio. Next, we calculate the overhead ratio of the on-line algorithm.

\subsection{Overhead Ratio of the On-Line Algorithm}

As we stated earlier, to focus on the benefits of the proposed algorithm, and simplify the analysis of the proposed algorithm, we assume that faults do not occur during checkpointing, and that the recovery time after a fault is zero. We also assume that the faults are detected immediately.

LEMMA 2. With the above assumptions, $R$, the average overhead ratio when the on-line algorithm for checkpointing placement is used, is given by

$$
R=\frac{\left(1-p_{2}\right) c_{1}+p_{2} c_{2}+\frac{e^{\lambda t_{1}}+\frac{\lambda p_{2}}{\lambda-\mu_{2}}\left(e^{\lambda t_{2}}-e^{\lambda t_{1}+\mu_{2}\left(t_{2}-t_{1}\right)}\right)-1}{\lambda}}{t_{1}+\frac{e^{\mu_{2}\left(t_{2}-t_{1}\right)}-1}{\mu_{2}} \cdot p_{2}}-1,
$$

where $p_{2}$ is the probability that a checkpoint is placed at $t_{2}$, given by

$$
p_{2}=\frac{\mu_{1}}{\mu_{1}+\mu_{2}} \cdot \frac{e^{\mu_{2} t_{1}}-e^{-\mu_{1} t_{1}}}{e^{\mu_{2} t_{2}}-e^{-\mu_{1} t_{1}}} .
$$

PrOOF. The proof of the lemma consists of the following propositions that derive the probability of placing a checkpoint at $t_{2}$ and $t_{1}$, the average length of an interval between checkpoints, and the average execution time of such interval.

Proposition 3. In a steady-state, $p_{2}$, the probability that the state size at a checkpoint is $s_{2}$ is

$$
p_{2}=\frac{\mu_{1}}{\mu_{1}+\mu_{2}} \cdot \frac{e^{\mu_{2} t_{1}}-e^{-\mu_{1} t_{1}}}{e^{\mu_{2} t_{2}}-e^{-\mu_{1} t_{1}}} .
$$

PROOF. In a steady-state, the probability that the state size at a checkpoint is $s_{2}$ satisfies the following equation

$p_{2}=\operatorname{Pr}\left\{\right.$ state size is $s_{2} \mid$ state size at previous cp was $\left.s_{2}\right\} \cdot p_{2}+$

$\operatorname{Pr}\left\{\right.$ state size is $s_{2} \mid$ state size at previous cp was $\left.s_{1}\right\} \cdot\left(1-p_{2}\right)$. (5)

The state size at a checkpoint is $s_{2}$ if, and only if, a checkpoint is placed at $t_{2}$, and a checkpoint is placed at $t_{2}$ if, and only if, the state size at $t_{1}$ is $s_{2}$ and the state size does not change in the interval $\left[t_{1}, t_{2}\right]$. Therefore,

$\operatorname{Pr}\left\{\right.$ state size is $s_{2} \mid$ state size at previous cp was $\left.s_{2}\right\}=$

$$
P_{2,2}\left(t_{1}\right) \cdot e^{-\mu_{2}\left(t_{2}-t_{1}\right)},
$$

and
$\operatorname{Pr}\left\{\right.$ state size is $s_{2} \mid$ state size at previous cp was $\left.s_{1}\right\}=$

$$
P_{1,2}\left(t_{1}\right) \cdot e^{-\mu_{2}\left(t_{2}-t_{1}\right)},
$$

where $P_{1,2}\left(t_{1}\right)$ and $P_{2,2}\left(t_{1}\right)$ are the transition probabilities from states $s_{1}$ and $s_{2}$, respectively, to $s_{2}$ at time $t_{1}$ given by

$$
\begin{aligned}
& P_{1,2}\left(t_{1}\right)=\frac{\mu_{1}}{\mu_{1}+\mu_{2}}\left(1-e^{-\left(\mu_{1}+\mu_{2}\right) t_{1}}\right), \\
& P_{2,2}\left(t_{1}\right)=\frac{\mu_{1}}{\mu_{1}+\mu_{2}}+\frac{\mu_{2}}{\mu_{1}+\mu_{2}} e^{-\left(\mu_{1}+\mu_{2}\right) t_{1}} .
\end{aligned}
$$

Assigning these values to (5) and solving for $p_{2}$ yields (4).

Note that $p_{2}$ is less than or equal to the steady-state probability of $s_{2}$, and it can be close to zero for high $\mu_{2}$. It means that the proposed algorithm uses the cheaper checkpoint more often than algorithms that do not consider the current checkpointing cost.

PROPOSITION 4. In a steady-state, $p_{1}$, the probability that a checkpoint is placed at $t_{1}$ is

$$
p_{1}=1-p_{2} e^{\mu_{2}\left(t_{2}-t_{1}\right)} .
$$

PROOF. A checkpoint is placed at $t_{2}$ if, and only if, it was not placed at $t_{1}$ and the state size remained $s_{2}$ in the interval $\left[t_{1}, t_{2}\right]$. Therefore,

$$
p_{2}=\left(1-p_{1}\right) \cdot e^{-\mu_{2}\left(t_{2}-t_{1}\right)},
$$

or

$$
p_{1}=1-p_{2} e^{\mu_{2}\left(t_{2}-t_{1}\right)} .
$$

COROLlary 5. The probability density function ( $p d f$ ) of the interval length $f(t)$ is

$$
\begin{aligned}
f(t)= & p_{1} \cdot \delta\left(t-t_{1}\right)+p_{2} \cdot \delta\left(t-t_{2}\right)+\left(1-p_{1}\right) \mu_{2} e^{-\mu_{2}\left(t-t_{1}\right)} . \\
& \left(U\left(t-t_{1}\right)-U\left(t-t_{2}\right)\right),
\end{aligned}
$$

where $\delta(\cdot)$ and $U(\cdot)$ are the impulse and step functions, respectively.

PROPOSITION 6. The average length of an interval between checkpoints is

$$
\overline{t_{i}}=t_{1}+\frac{e^{\mu_{2}\left(t_{2}-t_{1}\right)}-1}{\mu_{2}} \cdot p_{2} .
$$

ProOF. Let $f(t)$ be the probability density function ( $p d f)$ of the interval length, then

$$
\begin{aligned}
\overline{t_{i}} & =\int_{t_{1}}^{t_{2}} t f(t) d t \\
& =p_{1} \cdot t_{1}+p_{2} \cdot t_{2}+\left(1-p_{1}\right) \int_{t_{1}}^{t_{2}} t \mu_{2} e^{-\mu_{2}\left(t-t_{1}\right)} d t \\
& =t_{1}+\frac{e^{\mu_{2}\left(t_{2}-t_{1}\right)}-1}{\mu_{2}} \cdot p_{2} .
\end{aligned}
$$

PROPOSITION 7. The average execution time of an interval between checkpoints is 
$\bar{T}_{i}=\left(1-p_{2}\right) c_{1}+p_{2} c_{2}+\frac{e^{\lambda t_{1}}+\frac{\lambda p_{2}}{\lambda-\mu_{2}}\left(e^{\lambda t_{2}}-e^{\lambda t_{1}+\mu_{2}\left(t_{2}-t_{1}\right)}\right)-1}{\lambda}$

PROOF. Let $T(t, c(t))$ be the average execution time of an interval of length $t$ with checkpoint of cost $c(t)$ at the end of it. From Proposition 1, we know that

$$
T(t, c(t))=\frac{e^{\lambda t}-1}{\lambda}+c(t),
$$

and

$$
\begin{aligned}
\bar{T}_{i} & =\int_{t_{1}}^{t_{2}} T(t, c(t)) f(t) d t \\
& =p_{1} \cdot T\left(t_{1}, c_{1}\right)+p_{2} \cdot T\left(t_{2}, c_{2}\right)+\left(1-p_{1}\right) \int_{t_{1}}^{t_{2}} T\left(t, c_{1}\right) \mu_{2} e^{-\mu_{2}\left(t-t_{1}\right)} d t \\
& =\left(1-p_{2}\right) c_{1}+p_{2} c_{2}+\frac{e^{\lambda t_{1}}+\frac{\lambda p_{2}}{\lambda-\mu_{2}}\left(e^{\lambda t_{2}}-e^{\lambda t_{1}+\mu_{2}\left(t_{2}-t_{1}\right)}\right)-1}{\lambda} .
\end{aligned}
$$

PROPOSITION 8. The average overhead ratio of a program is

$$
R=\frac{\bar{T}_{i}}{\bar{t}_{i}}-1
$$

PROOF. To calculate the overhead ratio of a program, it is not enough to calculate the average overhead of an interval. We need to consider also the length of the intervals, since longer intervals occupy more of the program, and, thus, they have bigger influence on the overhead ratio. Therefore, using similar arguments to those used when considering the current life of a random point in time in renewal theory [6], the average overhead ratio of a program is given by

$$
\begin{aligned}
R & =\frac{1}{\bar{t}_{i}} \int_{t_{1}}^{t_{2}} \frac{T(t, c(t))-t}{t} t f(t) d t \\
& =\frac{\int_{t_{1}}^{t_{2}}(T(t, c(t))-t) f(t) d t}{\overline{t_{i}}} \\
& =\frac{\bar{T}_{i}}{\overline{t_{i}}}-1 .
\end{aligned}
$$

Assigning the values of $\bar{t}_{i}$ from (8) and $\bar{T}_{i}$ from (9) into the expression of the overhead ratio of a program given in (10) yields the expression in (3) and completes the proof of Lemma 2.

Given $\lambda, \mu_{1}, \mu_{2}, c_{1}$, and $c_{2}$, we can numerically find the values of $t_{1}$ and $t_{2}$ that minimize the overhead ratio $R$. More on the selection of $t_{1}$ and $t_{2}$ can be found in Section 4 .

\section{Comparison With Existing Algorithms}

To illustrate how the current knowledge about the cost of checkpointing and the proposed on-line algorithm can be used in reducing the execution time of a program, we compare the overhead ratio of a program using the on-line algorithm to the overhead ratio when the two strategies described in Section 2 are used, namely, the fixed intervals strategy and the optimal placement. The comparison to the fixed interval placement illustrates how the current knowledge about the cost of checkpointing helps to reduce the average execution time of a program. It also provides insight to the optimal values of $t_{1}$ and $t_{2}$ that minimize the overhead ratio. The comparison to the optimal placement strategy shows how much the performance of the on-line algorithm can be improved when the cost of checkpoints in all possible locations is known in advance and how the online and optimal algorithms differ in the placement of checkpoints.

The comparison of the new on-line algorithm with the fixed intervals placement strategy is done by comparing the overhead ratio of the on-line algorithm, given in Lemma 2, with the overhead ratio of the fixed intervals placement strategy, given in (2). The values of $t_{1}$ and $t_{2}$ for the on-line algorithm and the interval length $t$ for the fixed intervals placement strategy are those that minimize the overhead ratio.

Since we cannot analytically find the overhead ratio of the optimal placement, we used experimental results to compare the on-line placement algorithm with the optimal placement. We generated a large number of instances of the program's state size according to the two states Markov chain. For each such instance, we found the placement of the checkpoints when the optimal algorithm and the on-line algorithm are used. After the checkpoints were placed, we calculated the overhead ratio of the instance when both algorithms are used. Finally, we calculated the average overhead ratio over all instances that used the same parameters $\left(\lambda, \mu_{1}, \mu_{2}\right)$. The experimental values of the overhead ratio for the on-line algorithm are identical to the analytical values obtained using Lemma 2.

\subsection{Checkpointing with Fixed Intervals}

In Fig. 2, the overhead ratio of a program as a function of the fault rate $\lambda$ is shown. The figure shows the execution time when the checkpointing costs are $c_{1}=0.0005$ and $c_{2}=$ 0.005. The figure shows the execution time for two cases of $\mu_{1}$ and $\mu_{2}$. In Fig. $2 a, \mu_{1}=\mu_{2}=10$, and, in Fig. $2 b, \mu_{1}=\mu_{2}=1$. The figure compares the execution time of a program when fixed equidistant intervals are used to the execution time when the on-line algorithm for placement of checkpoints is used. As a reference, the figure also shows the overhead ratio if the cost of the checkpoints is only $c_{1}$. It can be seen in the figure that the on-line algorithm has a lower overhead ratio.

To understand why the on-line algorithm has a lower overhead ratio than the fixed interval placement, let's consider two extreme cases; the first is when the rate of changes in the state size is very low, and the second, when the rate of changes is very high.

When the rate of changes in the state size is very low, the probability of a change in the state size between $t_{1}$ and $t_{2}$ is practically zero, and checkpoints are placed only at $t_{1}$ and $t_{2}$. In this case, by using the optimal checkpointing intervals when the cost of a checkpoint is only $c_{1}$ or $c_{2}$ as $t_{1}$ and $t_{2}$, respectively, the on-line algorithm adapts to the current checkpoint cost, and uses the optimal interval for that cost. Therefore, for low rate of changes in the state size, the 


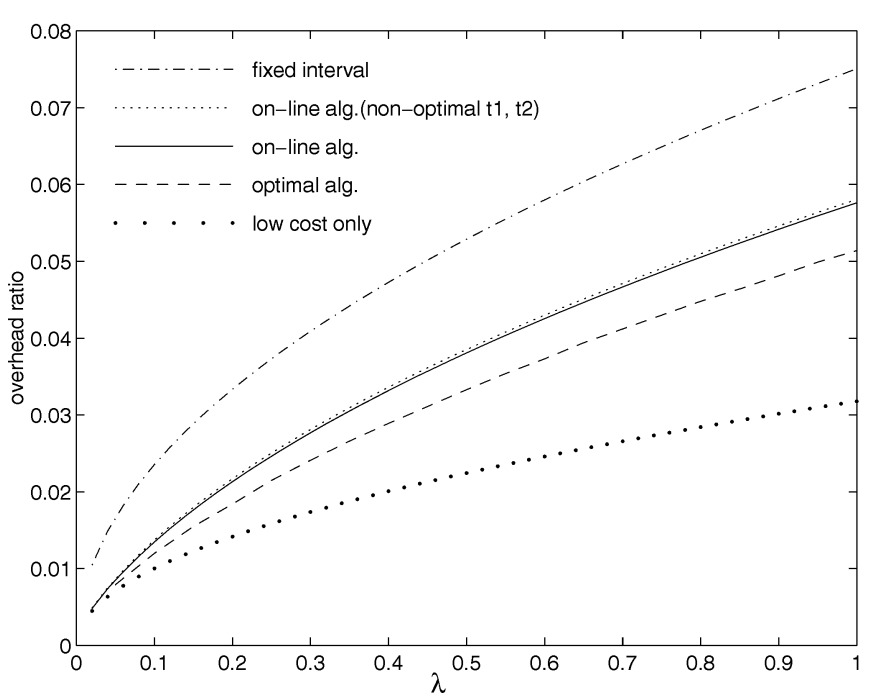

(a) $\mu_{1}=\mu_{2}=10$

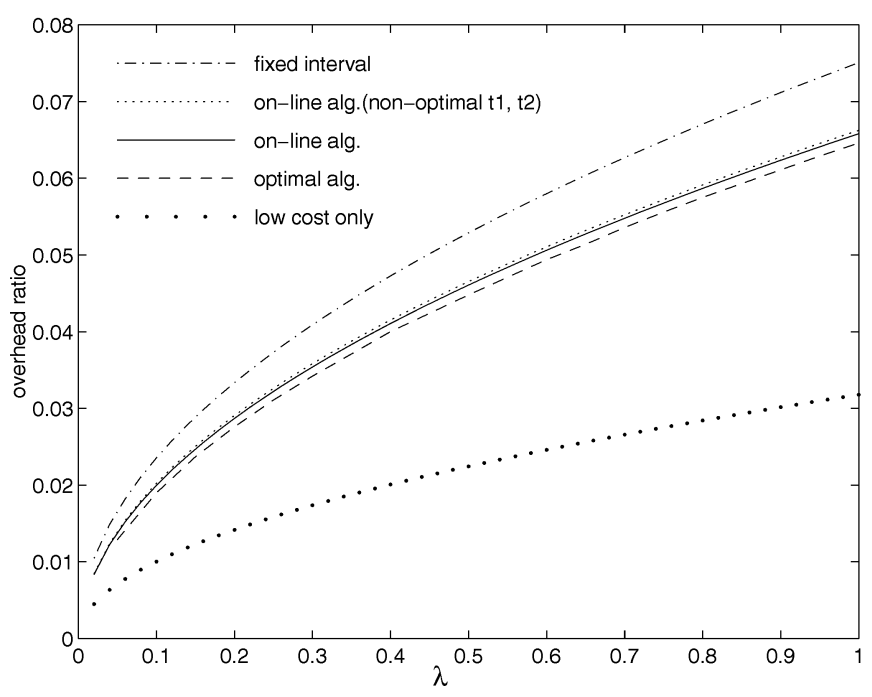

(b) $\mu_{1}=\mu_{2}=1$

Fig. 2. Overhead ratio as a function of $\lambda$.

optimal values of $t_{1}$ and $t_{2}$ are $t_{1, \text { opt }}=\tilde{t}_{1}$ and $t_{2, \text { opt }}=\tilde{t}_{2}$, where $\tilde{t}_{1}$ and $\tilde{t}_{2}$ are the optimal checkpointing intervals when the cost of checkpoints are the constants $c_{1}$ and $c_{2}$, respectively.

When the rate of changes in the state size is high, the online algorithm uses this fact to locate a point with a low cost near $\widetilde{t}_{1}$ and place a checkpoint at that point. The result is that the cost of a checkpoint is always the low cost, and the interval between the checkpoints is close to the optimal interval for that cost. In this case, it is always better to wait for a point with a low cost, and the optimal value for $t_{2}$ is very high.

In the medium range, when $\frac{1}{\mu_{2}}$ has the same order of magnitude as $\tilde{t}_{1}$, the on-line algorithm can take advantage of the points with low checkpointing cost that are near $\widetilde{t}_{1}$. To be sure that such points are not missed, the algorithm
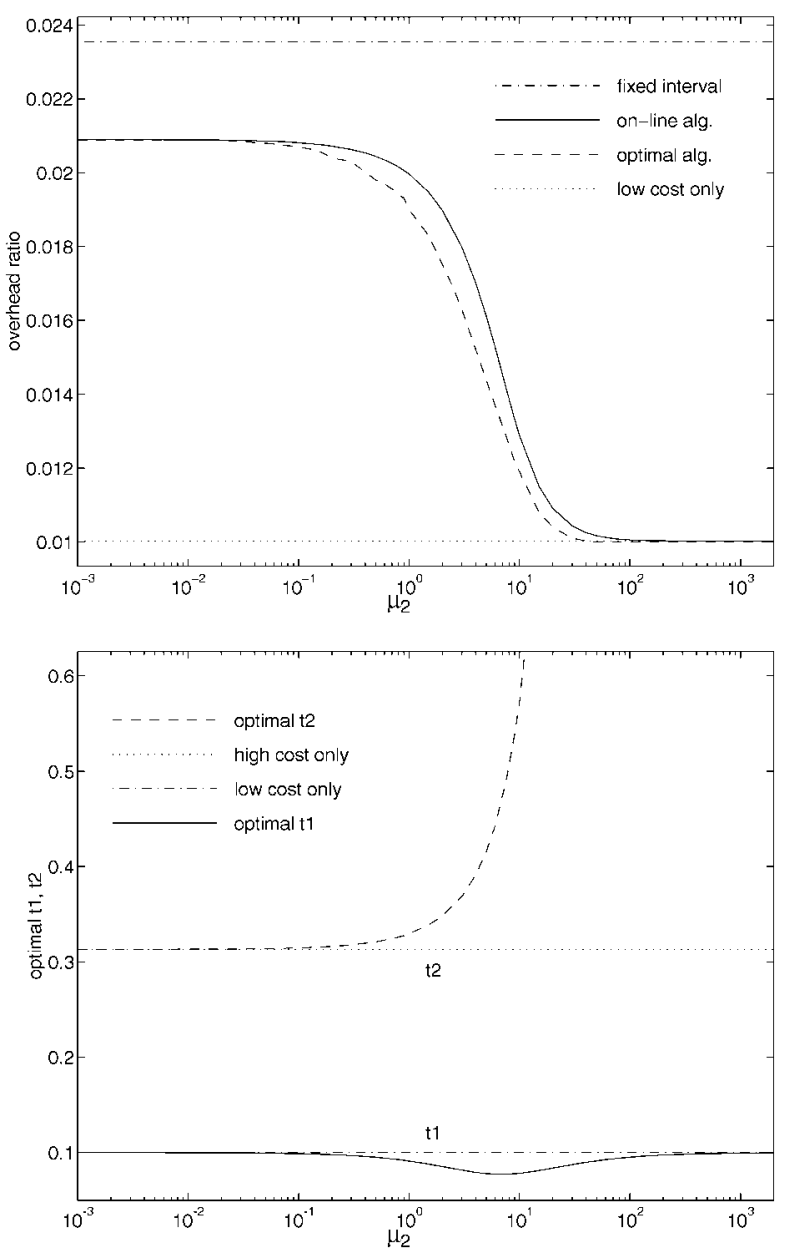

Fig. 3. Overhead ratio as a function of $\mu_{2}$.

starts to look for it before the optimal interval $\tilde{t}_{1}$. Therefore, for this range of $\mu_{2}, t_{1, \text { opt }}<\tilde{t}_{1}$. In this range, there is a good chance that the state size is going to change from high to low when $\tilde{t}_{2}$ is reached and that this change will occur fast enough so that it is better to wait for that change, and, therefore, in that range, $t_{2, \text { opt }}>\tilde{t}_{2}$.

Fig. 3 shows the overhead ratio and the optimal $t_{1}$ and $t_{2}$ as a function of $\mu_{2}$. The figure shows these values when $\lambda=0.1$, and the possible costs of a checkpoint are $c_{1}=0.0005$ and $c_{2}=0.005$ and $\mu_{1}=\mu_{2}$. The figure shows that, for low $\mu_{2}$, the overhead ratio is somewhat lower than the overhead ratio when fixed intervals are used. As $\mu_{2}$ increases, the overhead ratio of the on-line algorithm drops, and, for $\mu_{2}>100$, the overhead ratio is as if the cost of checkpointing was $c_{1}$ everywhere. The plot of the optimal $t_{1}$ and $t_{2}$ shows that, for low $\mu_{2}$, the optimal values equal to $\tilde{t}_{1}$ and $\tilde{t}_{2}$. When $\mu_{2}$ increases, the optimal value of $t_{1}$ decreases so that a point of low cost near $\tilde{t}_{1}$ is not missed, while the optimal value for $t_{2}$ increases to enable the algorithm to catch points with low cost at that area. Further increasing $\mu_{2}$ causes the optimal $t_{1}$ to 
increase and be closer to $\widetilde{t}_{1}$ because, for these values of $\mu_{2}$, the chance of finding a point with low cost is getting higher. The behavior of the overhead ratio and the optimal $t_{1}$ and $t_{2}$ for different ratios of $\mu_{2} / \mu_{1}$ are similar to the behavior shown in Fig. 3.

\subsection{Optimal Placement Strategy}

Fig. 2 shows the overhead ratio of a program as a function of the fault rate $\lambda$ for the on-line algorithm and optimal placement strategy. The figure shows that the optimal placement strategy performs better than the on-line algorithm, but the difference between them is not large, and the on-line algorithm is closer to the optimal placement strategy than the fixed intervals strategy.

In Fig. 3, the overhead ratio of the program, as a function of the rate of changes in the state size, is shown for both algorithms. The figure shows that both algorithms are affected in the same way by $\mu_{2}$. When $\mu_{2}$ is low, both algorithms adapt to the current state size and use the optimal interval for that state size. When $\mu_{2}$ is high, both algorithms can find points with small state size close to the optimal interval for that state size and place checkpoints there. Therefore, the overhead ratio is the same as if only the low state size exists. In the medium range for $\mu_{2}$, the optimal algorithm can use its knowledge about the cost of future possible checkpoints to achieve lower overhead ratio.

To understand the difference and similarities in checkpointing placement between the two algorithms, we examined a few of the instances of the random state sizes we generated, and looked where each of the algorithms placed its checkpoints. Fig. 4 shows three such instances. In all three cases, the fault rate is $\lambda=0.1$ and the checkpointing costs are $c_{1}=0.0005$ and $c_{2}=0.005$. In the top and bottom plots, $\mu_{1}=\mu_{2}=10$, and, in the middle plot, $\mu_{1}=\mu_{2}=3$. The plots show the state size of the program as a function of the time $t$, and the points where each of the algorithms places the checkpoints. The figure also shows the checkpointing placement of a modified version of the on-line algorithm, with rise detection in the state size, that is described later in the paper, in Section 5.

The top plot shows that both algorithms avoid placing checkpoints when the cost is high, even when there are long intervals of high cost. The difference in the algorithms in this plot is the interval between the checkpoints. The optimal algorithm knows exactly the intervals of low and high cost, so it can use them to place the checkpoints with the optimal interval between them. On the other hand, the on-line algorithm does not know when the cost is going to change from low to high, and so it prefers to use intervals which are shorter than the optimal interval when the cost is low, instead of losing the possibility to place a checkpoint with a low cost.

The second plot gives an example where the on-line algorithm places a checkpoint with high cost, while the optimal algorithm avoids the high cost interval. The optimal algorithm knows the length of the high cost interval, and that it is better not to place a checkpoint in it. On the other hand, the on-line algorithm anticipates that the interval is going to be much longer (because of the value of $\mu_{2}$ ), and, therefore, it
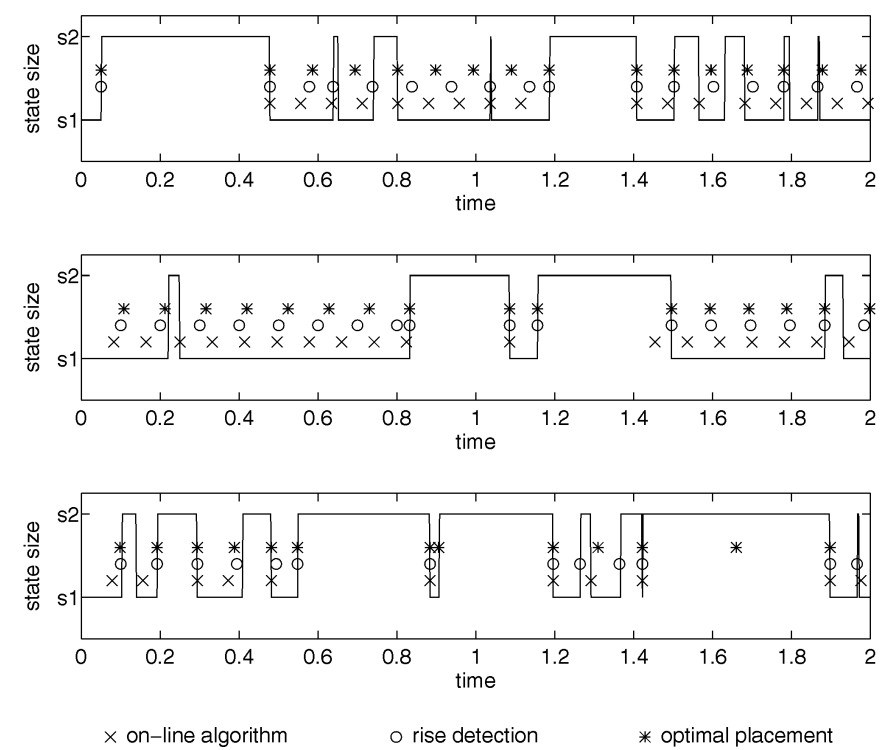

Fig. 4. Placement of checkpoints by the optimal off-line algorithm and the on-line algorithm with and without rise detection.

concludes that it is better to place a checkpoint in it.

The bottom plot shows an example where the optimal algorithm places a checkpoint at a point with a high cost, while the on-line algorithm avoids it. In this example, the on-line algorithm anticipates a fast change in the state size, and, therefore, it decides to wait for the small state size and place the checkpoint there. On the other hand, the optimal algorithm knows that the interval is going to be long, and, therefore, it is better to place a checkpoint in it.

\section{Detection of Increase in the State Size}

So far, we have assumed that the program does not have any knowledge about future changes in its state size. While this assumption is generally true, there are some cases when a partial knowledge about the future behavior exists. This partial knowledge can be used to improve the placement strategy. The simplest example about future knowledge is knowledge about changes in the state size just before they occur. When the memory allocation or deallocation functions are called, the program knows that state size is going to change before the change actually occurs.

Detection of changes in the state size before they occur is important when the state size increases. In this case, it might be beneficial to place a checkpoint with lower cost just before the state size increases. The ability to place a checkpoint just before the state size increases can contribute to the performance of the placement strategy in two ways. When the algorithm can place a checkpoint before the state size increases, it does not have to be "over-eager" when looking for points with low cost (the drop down in the value of $t_{1, \mathrm{opt}}$ in Fig. 3). Instead, it can wait until $\tilde{t}_{1}$ is reached, or the state size is about to change, and place the checkpoint at that time. Also, when a checkpoint is placed before the state size increases, the probability of placing a checkpoint with a large state size gets lower, and, thus, the checkpointing overhead is smaller. 
In this section, we show how to modify the on-line algorithm we presented in Section 3 to include the case of detection of an increase in the state size before they occur. We also show how to analyze the modified algorithm and compare its performance to the original on-line algorithm and optimal off-line placement.

\subsection{The Modified Algorithm}

In the modified algorithm, we add another point in time $t_{0}$, such that $t_{0} \leq t_{1}$. A checkpoint is placed at time $t, t_{0} \leq t<t_{1}$, if the state size at $t$ is $s_{1}$ and the state size at $t^{+}$is $s_{2}$. In other words, if the state size is changing from $s_{1}$ to $s_{2}$ during the interval $\left[t_{0}, t_{1}\right)$, then a checkpoint is placed just before the change. If a checkpoint is not placed in the interval $\left[t_{0}, t_{1}\right)$, then the algorithm continues as the algorithm in Section 3.

The analysis of the modified algorithm is essentially the same as the analysis of the original on-line algorithm that was shown in Lemma 2. The details of the analysis of the modified algorithm can be found in [13].

Fig. 5 shows the overhead ratio and the optimal $t_{0}$ and $t_{1}$ as a function of $\mu_{2}$. The figure shows this value when $\lambda=0.1$, the possible costs of a checkpoint are $c_{1}=0.0005$ and $c_{2}=$ 0.005 , and $\mu_{1}=\mu_{2}$.

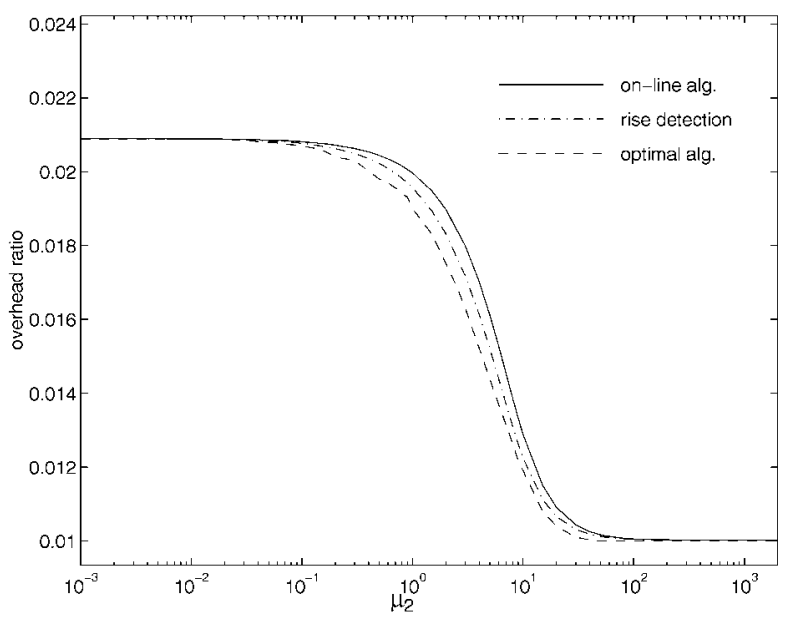

(a) Overhead ratio

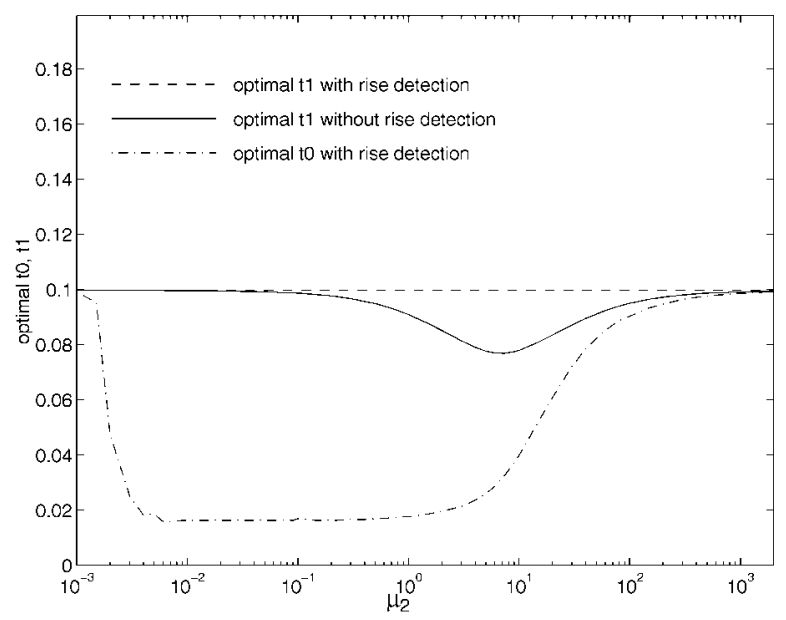

(b) Optimal Intervals
Fig. 5a shows the overhead ratio as a function of $\mu_{2}$ for the modified algorithm, the original on-line algorithm and the optimal off-line algorithm. The figure shows that the modified algorithm has a lower overhead ratio than the original on-line algorithm, and its behavior is closer to the optimal algorithm.

One of the reasons the modified algorithm performs better than the original on-line algorithm, is that it does not have to be "over eager" when looking for points with a small state size. The original algorithm does not know when the state size is going to increase. Therefore, in order not to lose the small state size, it places checkpoints before the optimal interval for the small state size $\tilde{t}_{1}$ is reached. On the other hand, the modified algorithm can wait until just before the state size changes or $\widetilde{t}_{1}$ is reached before it places a checkpoint, because it knows about the change in the state size before it occurs. Also, when the algorithm knows that the state size is going to increase, it is sometimes better to place a checkpoint after a short interval, especially when the $\mu_{2}$ is low. Therefore, the optimal value for $t_{0}$ for the modified algorithm is lower than the optimal value for $t_{1}$ in the original algorithm.

Fig. $5 \mathrm{~b}$ shows the optimal values of $t_{0}$ and $t_{1}$ for the modified algorithm as a function of $\mu_{2}$, and for comparison the optimal value of $t_{1}$ for the original algorithm. The figure confirms that the optimal value of $t_{1}$ for the modified algorithm is equal to $\tilde{t}_{1}$, and the drop in the value of $t_{1, \mathrm{opt}}$ that occur in the original algorithm to avoid losing points with a small state size is not needed in the modified algorithm. The figure also shows that, for low values of $\mu_{2}$, when the average time before the state size changes from $s_{2}$ to $s_{1}$ is high, it is beneficial to place checkpoints with a very short interval between them to use the small state size. As the value of $\mu_{2}$ gets higher, the value of $t_{0}$ is also getting higher, until it reaches $t_{1}$ for very high values of $\mu_{2}$.

The placement examples that are shown in Fig. 4 also help to illustrate the advantages of the modified algorithm over the original algorithm. The plots in the figure show three instances of changes in the state size, and the points where the on-line algorithm, the modified on-line algorithm, and the optimal algorithm placed their checkpoints. The figure shows that, during long periods of small state size, the modified algorithm places its checkpoints with the same intervals as the optimal algorithm, while the original algorithm uses smaller intervals. Another advantage that the modified algorithm has on the original algorithm is that it can sometimes avoid checkpoints with large state size, as can be seen in the middle plot of Fig. 4. Because the modified algorithm places checkpoints just before the state size increases, the probability that the state size will not change to $s_{1}$ before $t_{2}$ is smaller than the same probability in the original algorithm that places the checkpoint some time before the state size increases.

Fig. 5. Overhead ratio and optimal $t_{0}$ and $t_{1}$ for the modified algorithm. 


\section{A More Realistic Model}

The algorithm presented in this paper assumes that the program has only two state sizes and that the state size of the program is changing according to a Markov process with known parameters. In practice, both assumptions are not valid. The state size of a program is a continuous random process whose parameters are hard to estimate.

To overcome the continuous state size problem, two actions are needed. The first action is to quantize the state size, for example, to the nearest K-byte. If the quantization error is not big, the effects of the quantization on the performance of the algorithm are minimal. Finding a good quantization strategy that will minimize the effects on the performance of the algorithm and will not use too many quantization levels is still an open problem.

Even after quantization, a program is likely to have more than two state sizes. The algorithm presented in Section 3 can be extended to the case when there are more than two state sizes in the following way.

We assume that the possible state sizes are $s_{1}, s_{2}, \ldots, s_{n}$, and that the cost of a checkpoint for a state size $s_{i}$ is $c_{i}$, where $c_{1} \leq c_{2} \leq \cdots \leq c_{n}$. Each state size $s_{i}$ has an interval $t_{i}$ associated with it. The algorithm decides whether to place a checkpoint at time $t$, where $t$ is the time since the last checkpoint according to the following rules:

- If, at some time $t \in\left[t_{i}, t_{i+1}\right)$, the state size is $s_{1}, s_{2}, \ldots, s_{i}$, a checkpoint is placed at that time.

- At time $t=t_{n}$, a checkpoint is placed, regardless of the state size at that time.

The second unrealistic assumption made in the paper is the complete knowledge on the parameters of the random process that controls the state size of the program. These parameters are used to calculate the optimal values for the $t_{i}$ s. Without knowledge about these parameters, the optimal values have to be estimated. A good estimation for $t_{i, \mathrm{opt}}$ are the optimal intervals when the cost of checkpointing is a constant, $\tilde{t}_{i}$. The dotted line in Fig. 2 shows the overhead ratio when $\tilde{t}_{1}$ and $\tilde{t}_{2}$ are used instead of $t_{1, \mathrm{opt}}$ and $t_{2, \mathrm{opt}}$. As can be seen in the figure, the overhead ratio is almost identical. Since $\tilde{t}_{i}$ are independent of the parameters of the Markov process, they can be used even if these parameters are not known.

Another unrealistic assumption that was made in the paper was the time to roll back after a fault is detected. This assumption is not necessary for the operation of the algorithm. This assumption also does not affect the behavior of the algorithm. The only reason the assumption was made, was to simplify the analysis of the algorithm.

\section{Conclusions}

In this paper, we showed that knowledge about the current state size of the program can be used in placement of checkpoints in a program, and that using this knowledge can lead to a significant reduction in the overhead ratio. To illustrate how this knowledge can be used, we presented a new on-line algorithm for placement of checkpoints. The algorithm first tries to place a checkpoint in places where the cost of the checkpoint is small. Only if no such point was found, a checkpoint is placed at a point with higher checkpointing cost.

We studied the overhead ratio of a program using this algorithm, and compared the performance of the proposed algorithm to a simple algorithm that places the checkpoints at fixed intervals, and to the optimal placement strategy that uses a perfect a priori knowledge on the cost of checkpoints at all possible locations. The comparison results show that the proposed algorithm performs better than the fixed intervals algorithm, and a significant reduction of up to 66 percent in the overhead ratio can be obtained. Although the proposed algorithm uses only the cost of a checkpoint at the current location, its behavior is close to the optimal algorithm that uses an a priori knowledge of the checkpointing cost in all possible locations.

The same on-line placement strategy can be combined with other placement algorithms and improve their performance when the fault rate in the system is not a constant or when the changes in the state size do not occur according to a Markov process.

An interesting problem is to combine the on-line algorithm with some partial knowledge about the state size of the program in the future, like the information collected by the CATCH tool [9]. This additional knowledge about the state size can be used to improve the decision about the placement of checkpoints, and bring the algorithm closer to the optimal algorithm.

\section{ACKNOWLEDGMENTS}

The research reported in this paper was supported in part by U.S. National Science Foundation Young Investigator Award CCR-9457811, by the Sloan Research Fellowship, and by DARPA and BMDO through an agreement with NASA/OSAT.

\section{REFERENCES}

[1] A. Brock, "An Analysis of Checkpointing," ICL Technical J., vol. 1, 1979.

[2] K.M. Chandy and C.V. Ramamoorthy, "Rollback and Recovery Strategies for Computer Programs," IEEE Trans. Computers, vol. 21, no. 6, pp. 546-556, June 1972.

[3] E.G. Coffman and E.N. Gilbert, "Optimal Strategies for Scheduling Checkpoints and Preventive Maintenance," IEEE Trans. Reliability, vol. 39, pp. 9-18, Apr. 1990.

[4] A. Duda, "The Effects of Checkpointing on Program Execution Time," Information Processing Letters, vol. 16, pp. 221-229, June 1983.

[5] E. Gelenbe, "On the Optimum Checkpoint Interval," J. ACM, vol. 26, pp. 259-270, Apr. 1979.

[6] S. Karlin and H.M. Taylor, A First Course in Stochastic Processes. Academic Press, 1975.

[7] V.G. Kulkarni, V.F. Nicola, and K.S. Trivedi, "Effects of Checkpointing and Queueing on Program Performance," Comm. Statistics-Stochastic Models, vol. 6, pp. 615-648, Apr. 1990.

[8] P. L'Ecuyer and J. Malenfant, "Computing Optimal Checkpointing for Rollback and Recovery Systems," IEEE Trans. Computers, vol. 37, no. 4, pp. 491-496, Apr. 1988.

[9] C.-C. J. Li, E.M. Stewart, and W.K. Fuchs, "Compiler-Assisted Full Checkpointing," Software-Practice and Experience, vol. 24, pp. 871886, Oct. 1994.

[10] V.F. Nicola, "Checkpointing and the Modeling of Program Execution Time," Software Fault-Tolerance, M.R. Lyu, ed., pp. 167-188. John Wiley, 1995. 
[11] V.F. Nicola and J.M. van Spanje, "Comparative Analysis of Different Models of Checkpointing and Recovery," IEEE Trans. Software Eng., vol. 16, no. 8, pp. 807-821, Aug. 1990.

[12] S. Toueg and Ö. Babaoglu, "On the Optimum Checkpoint Selection Problem," SIAM J. Computing, vol. 13, pp. 630-649, Aug. 1984.

[13] A. Ziv, "Analysis and Performance Optimization of Checkpointing Schemes with Task Duplication," PhD thesis, Stanford Univ., 1995.

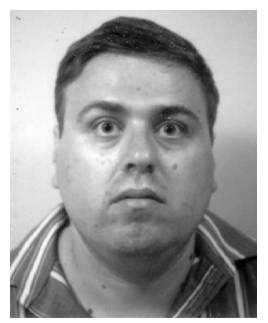

Avi Ziv received his BSc degree in computer engineering from the Technion, Israel, and his $\mathrm{MSc}$ and $\mathrm{PhD}$ in electrical engineering from Stanford University. He is currently a research fellow in the Haifa Research Laboratory, IBM Israel Science and Technology. His research interests include fault tolerant computing, parallel and distributed systems, and verification, testing, and reliability of hardware and software systems. $\mathrm{He}$ is a member of the IEEE and the IEEE Computer Society.

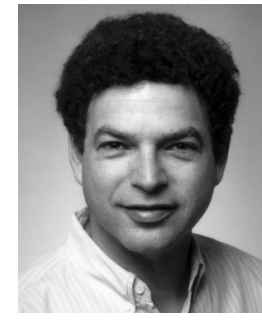

Jehoshua Bruck received the BSc and MSc degrees in electrical engineering from the Technion, Israel Institute of Technology, in 1982 and 1985 , respectively, and the $\mathrm{PhD}$ degree in electrical engineering from Stanford University in 1989.

Dr. Bruck is an associate professor of computation and neural systems and electrical engineering at the California Institute of Technology. His research interests include parallel and distributed computing, fault-tolerant computing, error-correcting codes, computation theory, and neural systems. $\mathrm{He}$ has extensive industrial experience, including serving as manager of the Foundations of Massively Parallel Computing Group at the IBM Almaden Research Center from 1990 to 1994, a research staff member at the IBM Almaden Research Center from 1989 to 1990, and a researcher at the IBM Haifa Science Center from 1982 to 1985.

Dr. Bruck is the recipient of a 1995 Sloan Research Fellowship, a 1994 U.S. National Science Foundation Young Investigator award, a 1992 IBM Outstanding Innovation award for his work on "Harmonic Analysis of Neural Networks," and a 1994 IBM Outstanding Technical Achievement award for his contributions to the design and implementation of the SP-1, the first IBM scalable parallel computer. He received six IBM Plateau Invention Achievement awards and he holds 19 patents. Dr. Bruck is a senior member of the IEEE. 